\title{
Optical properties of InGaN/GaN nanopillars fabricated by postgrowth chemically assisted ion beam etching
}

\section{AUTHOR(S):}

Kawakami, Y.; Kaneta, A.; Su, L.; Zhu, Y.; Okamoto, K.; Funato, M.; Kikuchi, A.; Kishino, K.

\section{CITATION:}

Kawakami, Y.... [et al]. Optical properties of InGaN/GaN nanopillars fabricated by postgrowth chemically assisted ion beam etching. JOURNAL OF APPLIED PHYSICS 2010, 107(2): 023522.

\section{ISSUE DATE:}

2010-01

URL:

http://hdl.handle.net/2433/147195

\section{RIGHT:}

Copyright 2010 American Institute of Physics. This article may be downloaded for personal use only. Any other use requires prior permission of the author and the American Institute of Physics. The following article appeared in JOURNAL OF APPLIED PHYSICS107, 023522 (2010) and may be found at 


\title{
Optical properties of InGaN/GaN nanopillars fabricated by postgrowth chemically assisted ion beam etching
}

\author{
Y. Kawakami, ${ }^{1,3, a)}$ A. Kaneta ${ }^{1,3}$ L. Su, ${ }^{1}$ Y. Zhu, ${ }^{1}$ K. Okamoto, ${ }^{1}$ M. Funato, ${ }^{1,3, b)}$ \\ A. Kikuchi, ${ }^{2,3}$ and K. Kishino ${ }^{2,3}$ \\ ${ }^{1}$ Department of Electronic Science and Engineering, Kyoto University, Kyoto 615-8510, Japan \\ ${ }^{2}$ Department of Electrical and Electronics Engineering, Sophia University, Tokyo 102-8554, Japan \\ ${ }^{3}$ CREST, JST, Saitama 332-0012, Japan
}

(Received 7 October 2009; accepted 30 November 2009; published online 27 January 2010)

\begin{abstract}
The optical properties of InGaN/GaN quantum wells, which were nanopatterned into cylindrical shapes with diameters of $2 \mu \mathrm{m}, 1 \mu \mathrm{m}$, or $500 \mathrm{~nm}$ by chemically assisted ion beam etching, were investigated. Photoluminescence (PL) and time-resolved PL measurements suggest inhomogeneous relaxation of the lattice-mismatch induced strain in the InGaN layers. By comparing to a strain distribution simulation, we found that partial stain relaxation occurs at the free side wall, but strain remains in the middle of the pillar structures. The strain relaxation leads to an enhanced radiative recombination rate by a factor of $4-8$. On the other hand, nonradiative recombination processes are not strongly affected, even by postgrowth etching. Those characteristics are clearly reflected in the doughnut-shape emission patterns observed by optical microscopy. () 2010 American Institute of Physics. [doi:10.1063/1.3280032]
\end{abstract}

\section{INTRODUCTION}

Extensive scientific and technological efforts have been devoted to optoelectronic devices based on III-nitride semiconductors, which have led to dazzling blue light-emitting diodes with a record external quantum efficiency of $75.5 \% .1$ Although the In composition in InGaN quantum well $(\mathrm{QW})$ active layers can control the emission wavelength, the emission efficiency drastically decreases beyond the blue-green spectral range. This so-called green gap issue is due to two major factors: the potential fluctuations and quantum confinement Stark effect (QCSE). Using scanning near field optical microscopy $(\mathrm{SNOM})^{2-4}$ and confocal laser scanning microscopy, ${ }^{5}$ we have investigated the emission mechanisms of InGaN QWs, and found that their optical properties strongly depend on submicron-scale potential fluctuations.

Quite recently, we have developed nanospectroscopy based on SNOM and atomic force microscopy. ${ }^{4}$ This newly developed technique to assess violet, blue, and green emitting QWs has revealed that in blue-emitting QWs, potential fluctuations prevent threading dislocations (TDs) from capturing carriers/excitons. In contrast in green-emitting QWs, potential fluctuations are associated with newly introduced TDs. Carriers/excitons with a radiative lifetime, $\tau_{\mathrm{r}}$, elongated by the QCSE easily diffuse to such TDs and recombine nonradiatively. Therefore, one promising way to increase the luminescence intensity in the green spectral range is to shorten the diffusion length of carriers/excitons, which corresponds to a reduction in $\tau_{\mathrm{r}}$. Thus, the stronger luminescence is a consequence of a better internal quantum efficiency, which is described as $\left(1+\tau_{\mathrm{r}} / \tau_{\mathrm{nr}}\right)^{-1}$, where $\tau_{\mathrm{nr}}$ is a nonradiative recombination lifetime.

Of the several proposals to promote radiative recombi-

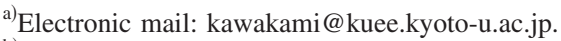

${ }^{b)}$ Electronic mail: funato@kuee.kyoto-u.ac.jp.
}

nation, such as nonpolar/semipolar $\mathrm{QWs}^{6-9}$ and plasmonics, ${ }^{10}$ nanostructures are one of the most promising approaches because the free side wall may relax the strain in QWs, and consequently, suppress the QCSE. ${ }^{11-19}$ In particular, InGaN/GaN nanocolumns, which are self-formed by molecular beam epitaxy, do not involve TDs ${ }^{11,12}$ and show a very fast $\tau_{\mathrm{r}}$ due to strain relaxation in InGaN QWs. ${ }^{16}$ To date, the sizes and positions of nanocolumns are well controlled by selective area growth, ${ }^{17}$ and the homogeneity has drastically been improved. ${ }^{18}$ On the other hand, nanorings and nanopillars are constructed from conventional planar QWs by postgrowth processes. ${ }^{13,14,19}$ This top-down approach can be advantageous over the above-mentioned bottom-up approach because arbitrary structures can be fabricated at arbitrary positions, allowing well-established growth on the (0001) plane. Moreover, this approach will pave the way toward controlled artificial spatial inhomogeneity in InGaN light-emitting layers, which was proposed in Ref. 5 as an effective way to improve emission efficiency and, furthermore, to create novel functionality. However, to date, radiative and nonradiative recombination processes have yet to be thoroughly studied for bottom-up and top-down nanostructures. Particularly for top-down nanostructures, the generation of nonradiative recombination centers by postgrowth processes is a major concern. Herein the details of the recombination dynamics in InGaN/GaN nanopillars fabricated from planar QWs by chemically assisted ion beam etching (CAIBE) are investigated. It is demonstrated that CAIBE does not generate a nonradiative pathway, which enables the intrinsic properties of nanopillars to be extracted. Optical characterizations and theoretical considerations revealed that the lattice-mismatch induced strain is relaxed around the free side wall, whereas it remains around the middle of nanopillars, which lead to their unique optical properties. 


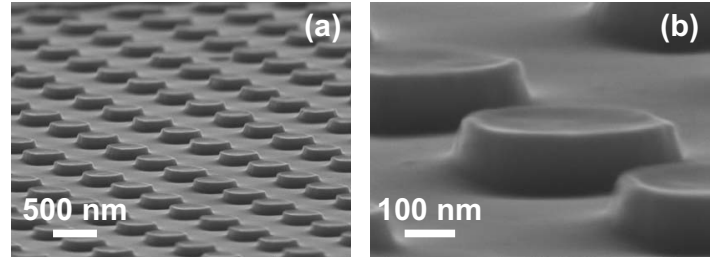

FIG. 1. (a) Bird's eye view of InGaN/GaN nanopillars observed by SEM and (b) the magnified image. Pillar diameter is $500 \mathrm{~nm}$.

\section{NANOPILLAR FABRICATION}

Initially, GaN was grown on a sapphire (0001) substrate using metalorganic vapor phase epitaxy. Then a 3-nm-thick InGaN single QW (SQW) and a 10-nm-thick GaN capping layer were grown on GaN. The In composition was $25 \%$, unless stated. Cylindrical nanopillars with diameters of $2 \mu \mathrm{m}, 1 \mu \mathrm{m}$, and $500 \mathrm{~nm}$ were fabricated ex situ. The patterns were defined into a 200-nm-thick polymethylmethacrylate resist spun onto the samples and exposed to a field emission electron beam lithography system. After lithography, the beam patterns were transferred into the samples using CAIBE. CAIBE employs both chemical and physical etching processes. Thus, anisotropic but fast etching is possible under gentle conditions. ${ }^{20}$ In this study, Xe was used for sputtering, while $\mathrm{Cl}_{2}$ was used for chemical etching. The etched depth was $150 \mathrm{~nm}$. Therefore, the side wall of the SQW was exposed to air. Figure 1 displays scanning electron microscopy (SEM) images of a fabricated nanopillar sample with a diameter of $500 \mathrm{~nm}$. It is confirmed that well-defined structures are fabricated even for this minimum diameter.

\section{RADIATIVE RECOMBINATION PROCESSES AFFECTED BY STRAIN RELAXATION AROUND THE FREE SIDE WALL}

\section{A. Optical characterizations}

Figure 2 shows a fluorescent microscopy image of nanopillars with a $2 \mu \mathrm{m}$ diameter acquired at room temperature (RT). The image was taken at the surface normal. Details of the emission pattern are discussed in Sec. V. As seen in Fig. 2, $8 \times 8$ nanopillars were fabricated within a $30 \times 30 \mu \mathrm{m}^{2}$ square region, which was surrounded by an unprocessed re-

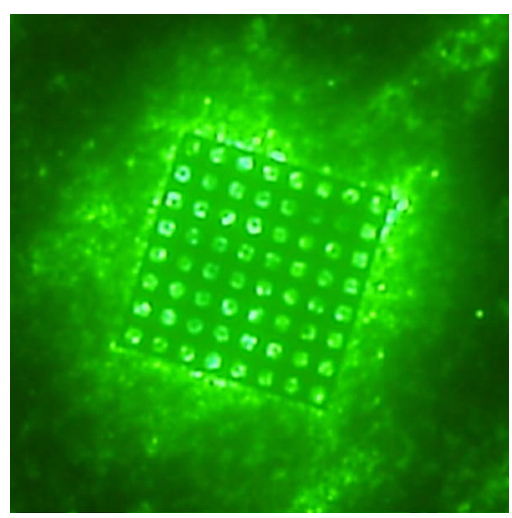

FIG. 2. (Color online) Fluorescent microscopy image observed at the surface normal of nanopillars. Image is acquired at RT and the pillar diameter is $2 \mu \mathrm{m}$.

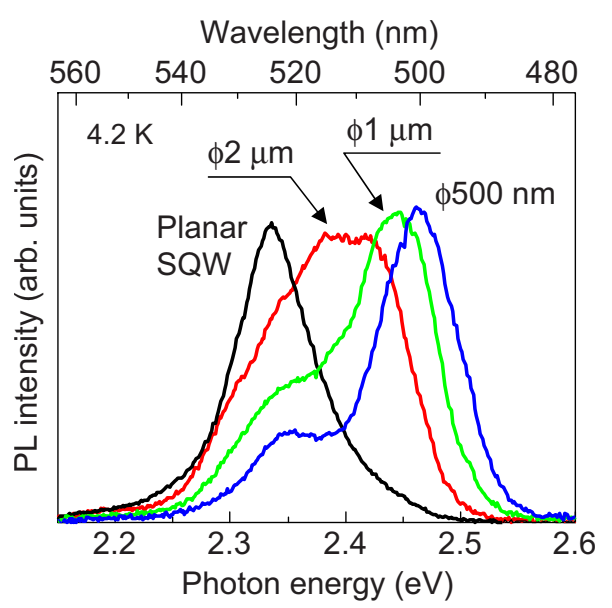

FIG. 3. (Color online) PL spectra of a planar SQW and nanopillars with diameters of $2 \mu \mathrm{m}, 1 \mu \mathrm{m}$, and $500 \mathrm{~nm}$ acquired at $4.2 \mathrm{~K}$.

gion involving the planar SQW. Obviously, the emission from the nanopillars was much stronger than that from the planar SQW, even though the nanopillar fabrication decreased the emission area. Similar trends were observed for nanopillars with different diameters. Contrary to conventional III-V semiconductors, such as GaAs, where postgrowth processes typically cause damage and degrade the emission properties, the external quantum efficiency was drastically improved for our InGaN/GaN nanopillars. This finding suggests that the surface recombination velocity of (In) GaN is very slow not only for the self-formed nanocolumns ${ }^{16}$ but also for the nanopillars. (The influence of the nanopillar fabrication on the nonradiative recombination rate is quantified in Sec. IV.) However, the mechanisms causing the striking difference between conventional III-V and nitride semiconductors have yet to be clarified.

The emission intensity is generally determined as the product of the internal quantum efficiency and light extraction efficiency. Therefore, to identify which factors play larger roles in the increased emission intensity observed in Fig. 2, photoluminescence (PL) measurements were performed at $4.2 \mathrm{~K}$ and RT under selective excitation by a frequency doubled Ti:sapphire laser emitting at $400 \mathrm{~nm}$. The excitation spots were adjusted using confocal microscopy in order to assess a single nanopillar while maintaining an excitation carrier density of $8.0 \times 10^{17} / \mathrm{cm}^{3}$. Figure 3 shows the PL spectra at $4.2 \mathrm{~K}$ with the emission intensities normalized by the emitting areas. The PL integrated intensities were nearly the same for all samples, indicating that there is not a difference in the light extraction efficiencies among the samples. This was supported by fluorescent microscopy performed at $4.2 \mathrm{~K}$, where the emission intensity was rather independent of the sample structures including the planar SQW. Our finite-difference time-domain (FDTD) simulation suggests that thicker GaN capping layers increase the light extraction efficiency, especially to the near-normal direction. Hence, the thin capping layer in the present nanopillar (10 $\mathrm{nm}$ ) did not affect the light extraction efficiency. At RT, on the other hand, compared to the planar SQW, the PL integrated intensities increased by factors of 3.3, 7.7, and 6.6 for 


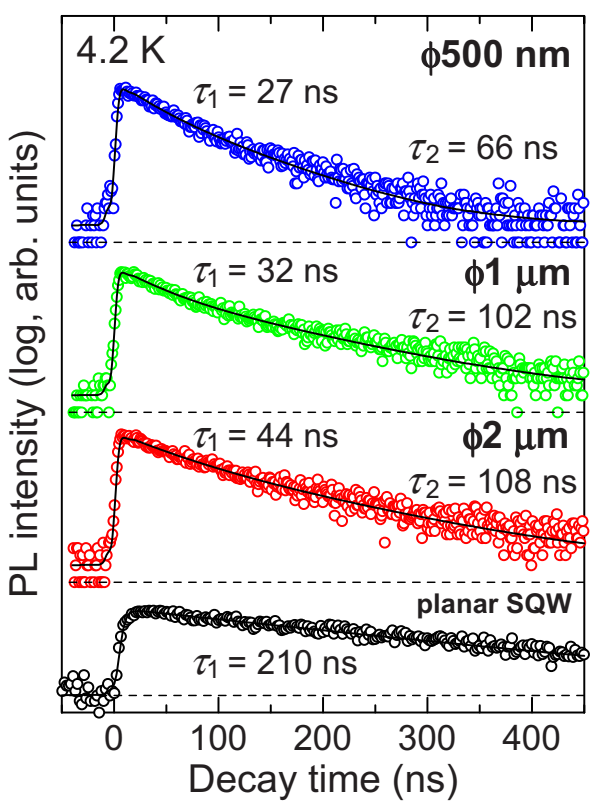

FIG. 4. (Color online) PL decay curves acquired at the PL peaks for planar SQW and nanopillars at $4.2 \mathrm{~K}$.

$\phi 2 \mu \mathrm{m}, \phi 1 \mu \mathrm{m}$, and $\phi 500 \mathrm{~nm}$ nanopillars, respectively. These increases can be attributed to the improved internal quantum efficiencies.

Figure 3 shows another interesting characteristic in the PL spectra. The PL of the planar SQW consisted of a single peak at $2.34 \mathrm{eV}$. However, nanopillar fabrication produced an additional peak, and smaller nanopillars shifted it toward higher energies. As deduced from the similar structure of self-formed nanocolumns, ${ }^{16}$ the nanopillar structure may relax the strain in the InGaN well layers. For fully strained $\mathrm{In}_{0.25} \mathrm{Ga}_{0.75} \mathrm{~N}$ on unstrained $\mathrm{GaN}$, the estimated internal electric field due to piezoelectric and spontaneous polarizations was $2.54 \mathrm{MV} / \mathrm{cm}$. If this electric field is completely eliminated, then the PL peak should shift from 2.34 to $2.70 \mathrm{eV}$. Thus, Fig. 3 indicates that the relaxation is not uniform, and that regions with different degrees of relaxation coexist within a nanopillar SQW.

To demonstrate the internal electric field reduced by the strain relaxation, the excitation density dependence of the PL peak position was assessed. It was found that the increase in the photogenerated carrier densities from $2 \times 10^{16}$ to 8 $\times 10^{18} / \mathrm{cm}^{3}$ caused blueshifts of $110,50,30$, and $10 \mathrm{meV}$ for the planar SQW, $\phi 2 \mu \mathrm{m}, \phi 1 \mu \mathrm{m}$, and $\phi 500 \mathrm{~nm}$ nanopillars, respectively. Because the samples were on the same wafer, the degree of state filling should be the same. Therefore, the observed decrease in the blueshift can be attributed to the reduced internal electric field, and the smaller blueshift for smaller nanopillars suggests that the partial strain relaxation is promoted by the size reduction.

Furthermore, the strain relaxation should increase the overlap between electron and hole wave functions, and consequently, heighten the radiative recombination rate. This assertion was confirmed by time-resolved PL (TRPL) measurements performed at $4.2 \mathrm{~K}$ for ensembles of nanopillars. The laser used was the same as that for the above experiments, but the excitation density was $6.7 \times 10^{16} / \mathrm{cm}^{3}$. Figure 4 shows the PL decays $[I(t)]$ at the PL peaks where the symbols are the experimental data and the lines are the results of fits with double exponential curves described by $I(t)$ $=A_{1} \exp \left(-t / \tau_{1}\right)+A_{2} \exp \left(-t / \tau_{2}\right) \cdot A_{1}$ and $\tau_{1}\left(A_{2}\right.$ and $\left.\tau_{2}\right)$ are for the faster (slower) decay component. The fitting errors in terms of the reduced $\chi$-square value were $2.0,6.0,3.7$, and 11.7 for the planar SQW, $\phi 2 \mu \mathrm{m}, \phi 1 \mu \mathrm{m}$, and $\phi 500 \mathrm{~nm}$ nanopillars, respectively. For the planar SQW, single exponential fit, that is, $A_{2}=0$, provided the best fit with $\tau_{1}$ $=210 \mathrm{~ns}$, which represented the radiative recombination lifetime. For the nanopillar samples, smaller pillars had faster decay components. (Note that the radiative recombination lifetime is the reciprocal of the radiative recombination rate.) Furthermore, it is interesting to compare the ratio of the two decay components: $A_{1} / A_{2}=1.2$ for $\phi 2 \mu \mathrm{m}, 1.5$ for $\phi 1 \mu \mathrm{m}$, and 2.0 for $\phi 500 \mathrm{~nm}$. That is, the faster decay, which had a lifetime of a few tens ns, became more dominant as the nanopillar diameter decreased. On the other hand, the slower decay times in the nanopillars are closer to the radiative lifetime of the planar SQW (210 ns). These findings led us to the conclusion that the faster decays of the nanopillars are due to radiative recombination in strain-relaxed regions but the slower decays are due to radiative recombination in strained regions. Hence, the decays reflect the inhomogeneous strain relaxation in the nanopillars suggested by PL in Fig. 3. The observed fast decay lifetimes of 44, 32, and $27 \mathrm{~ns}$ for the $\phi 2 \mu \mathrm{m}, \phi 1 \mu \mathrm{m}$, and $\phi 500 \mathrm{~nm}$ nanopillars indicate that the radiative recombination rates are enhanced by factor of 4.8, 6.6 , and 7.8, respectively, via the strain relaxation.

\section{B. Discussion}

To support the above experimental results, the polarization-induced electric field, the square overlap of the electron and hole wave functions, and the transition energy were calculated as a function of strain in 3-nm-thick $\mathrm{In}_{0.25} \mathrm{Ga}_{0.75} \mathrm{~N} / \mathrm{GaN} \mathrm{SQWs}$. It was assumed for simplicity that $\mathrm{GaN}$ is always unstrained, whereas $\operatorname{In}_{0.25} \mathrm{Ga}_{0.75} \mathrm{~N}$ can be strained irrespective of the lattice mismatch. Therefore, only the InGaN layers involve electric fields. The piezoelectric polarization was first evaluated as a function of strain, and then, the internal electric field was deduced with considering the contribution from the spontaneous polarization. The obtained internal electric field determined the band profile of the 3-nm-thick $\operatorname{In}_{0.25} \mathrm{Ga}_{0.75} \mathrm{~N} / \mathrm{GaN} \mathrm{SQW}$, for which electron and hole wave functions, their square overlap, and transition energy were calculated.

Figure 5 shows the results. The in-plane strain $\left(\epsilon_{x x}\right.$ $=\epsilon_{y y}$ ) for fully strained $\operatorname{In}_{0.25} \mathrm{Ga}_{0.75} \mathrm{~N}$ on $\mathrm{GaN}$ is $-2.65 \%$ and the closed circle in Fig. 5(a) denotes the corresponding square overlap. Because square overlaps of the electron and hole wave functions are inversely proportional to radiative lifetimes, this plot represents the radiative lifetime of the planar SQW (210 ns) and relates the experimentally estimated radiative lifetime to the calculated square overlap, as indicated by two vertical axes on the left hand side of Fig. 5(a). Then, from the experimentally observed variation of the radiative lifetimes due to the nanopillars (Fig. 4), the open circles in Fig. 5(a) are determined on the square overlap 


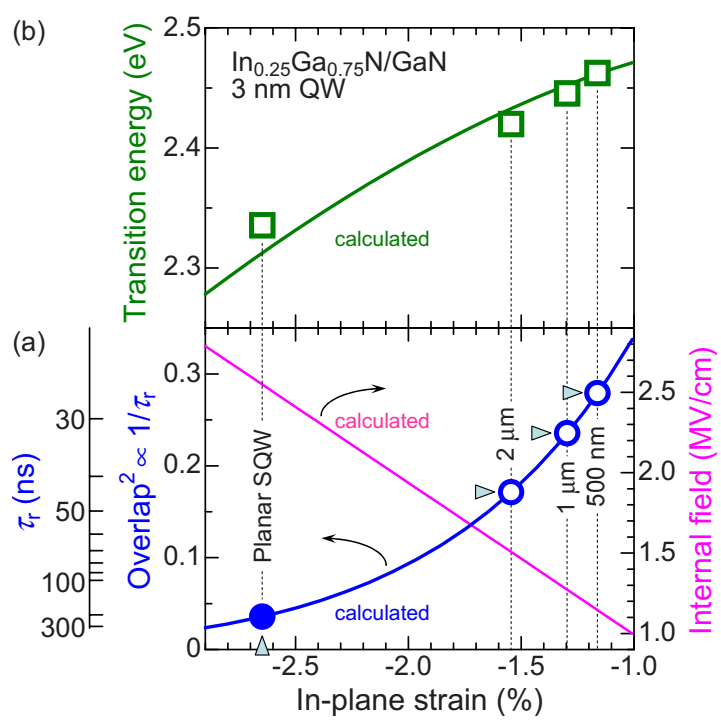

FIG. 5. (Color online) Variation in (a) square overlap between the electron and hole wave functions, internal electric field and (b) transition energy as a function of strain. In both (a) and (b), solid curves are the calculated results, while the symbols indicate experimental data. In (b), PL peak positions are used as transition energies.

curve, and correspondingly, the strains are estimated to be $-1.54 \%,-1.30 \%$, and $-1.16 \%$ for $\phi 2 \mu \mathrm{m}, \phi 1 \mu \mathrm{m}$, and $\phi 500 \mathrm{~nm}$ nanopillars, respectively.

Using the estimated strains, the calculated internal electric fields are $2.54,1.51,1.27$, and $1.15 \mathrm{MV} / \mathrm{cm}$ for the planar $\mathrm{In}_{0.25} \mathrm{Ga}_{0.75} \mathrm{~N} \mathrm{QW}, \phi 2 \mu \mathrm{m}, \phi 1 \mu \mathrm{m}$, and $\phi 500 \mathrm{~nm}$ nanopillars, respectively, which can also be confirmed in Fig. 5(a). The calculations quantify the partial strain relaxation, which was suggested in Fig. 3. The residual electric field should cause the QCSE. Figure 5(b) compares the calculated transition energy (solid curve) and PL peak positions (symbols). The calculation considers the exciton binding energies, which are $22 \mathrm{meV}$ for the planar SQW and $28 \mathrm{meV}$ for the $\phi 500 \mathrm{~nm}$ nanopillars. (How to calculate the exciton binding energy can be found in Ref. 21.) The calculation agrees well with the experimental data, supporting the current analysis for the strain and related optical properties.

\section{Simulation of strain distribution in InGaN/GaN nanopillars}

To support the above mentioned in-plane strain reduction, a simulation was performed by solving the strain tensor equation via the finite elemental method. Figure 6 shows the result for a 3-nm-thick $\mathrm{In}_{0.25} \mathrm{Ga}_{0.75} \mathrm{~N}$ with $200 \mathrm{~nm}$ pillar diameter. In the middle of the pillar, the in-plane strain $\left(\epsilon_{x x}\right.$ $\left.=\epsilon_{y y}\right)$ in InGaN is about $-2.6 \%$, which corresponds to fully strained $\mathrm{In}_{0.25} \mathrm{Ga}_{0.75} \mathrm{~N}$ on $\mathrm{GaN}$. On the other hand, the free side wall relaxes the strain to $-1.5 \%$ to $-1.0 \%$. It was confirmed that nanopillars with diameters larger than $200 \mathrm{~nm}$ do not change the degree of strain relaxation at the side wall, which is why we selected $\phi 200 \mathrm{~nm}$ for the calculation to reduce the calculation time without degrading the accuracy. As shown in Fig. 6, the strain distribution is not uniform, which is consistent with the PL and TRPL experiments (Figs. 3 and 4). Furthermore, the calculated degree of strain reduc-

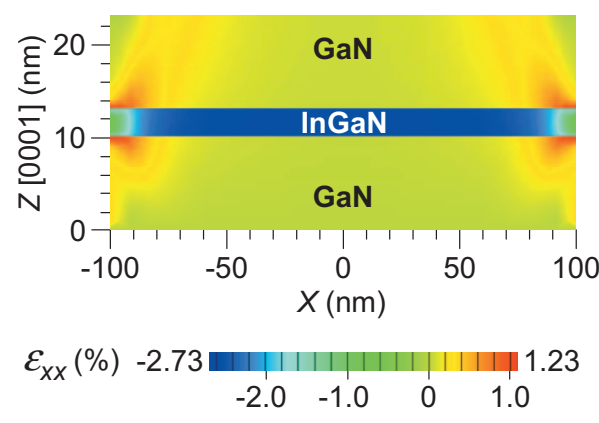

FIG. 6. (Color) In-plane strain calculation for 3-nm-thick $\operatorname{In}_{0.25} \mathrm{Ga}_{0.75} \mathrm{~N}$ pseudomorphically embedded in GaN nanopillar with a diameter of $200 \mathrm{~nm}$. $x$ is along the diameter.

tion at the side wall agrees quite well with the experimentally derived results from TRPL. Such an inhomogeneous strain distribution has previously been reported for GaN/ AlGaN and InGaN/GaN nanopillars. ${ }^{15,19}$ It is noteworthy that the enhanced radiative recombination rate at the side wall may cause doughnut-shape emission patterns, which are discussed in more detail in Sec. V

\section{RADIATIVE AND NONRADIATIVE RECOMBINATION AT RT: INFLUENCE OF POSTGROWTH NANOPILLAR FABRICATION}

To assess radiative and nonradiative recombination processes at RT, temperature dependence PL and TRPL measurements were performed. Assuming the internal quantum efficiency at low temperatures is nearly $100 \%$, the PL intensity ratio between $4.2 \mathrm{~K}$ and $\mathrm{RT}, I_{\mathrm{RT}} / I_{4.2 \mathrm{~K}}$, can be regarded as the internal quantum efficiency, $\left(1+\tau_{\mathrm{r}} / \tau_{\mathrm{nr}}\right)^{-1}$, at RT. This assumption seems valid because the PL intensity remained unchanged below $50 \mathrm{~K}$; if nonradiative processes are not negligible, then the PL intensity should be weakened by higher temperatures. On the other hand, the PL decay expressed as $\tau_{\mathrm{PL}}^{-1}=\tau_{\mathrm{r}}^{-1}+\tau_{\mathrm{nr}}^{-1}$ was measured at RT by TRPL. Using the experimentally obtained $I_{\mathrm{RT}} / I_{4.2 \mathrm{~K}}$ and $\tau_{\mathrm{PL}}$ at RT, then $\tau_{\mathrm{r}}$ and $\tau_{\mathrm{nr}}$ can be evaluated.

Table I summarizes the results. For all samples, the estimated radiative recombination lifetime $\left(\tau_{\mathrm{r}}\right)$ is longer than that at $4.2 \mathrm{~K}$ (Fig. 4), reflecting the temperature-dependence of radiative recombination of excitons in low-dimensional structures. Comparing all the samples indicates that the nanopillars shorten the radiative lifetimes; that is, the radiative recombination rates are heightened by factors of 1.9, 4.7, and 5.3 for the $\phi 2 \mu \mathrm{m}, \phi 1 \mu \mathrm{m}$, and $\phi 500 \mathrm{~nm}$ nanopillars, respectively. The enhancement factors differ slightly from

TABLE I. Summary of the temperature dependence PL and TRPL measurements. Intensity ratio between $4.2 \mathrm{~K}$ and $\mathrm{RT}\left(I_{\mathrm{RT}} / I_{4.2 \mathrm{~K}}\right)$ and decay time for TRPL at RT $\left(\tau_{\mathrm{PL}}\right)$ are experimental results, whereas $\tau_{\mathrm{r}}$ and $\tau_{\mathrm{nr}}$ are calculated.

\begin{tabular}{lcccc}
\hline \hline & $\begin{array}{c}I_{\mathrm{RT}} / I_{4.2 \mathrm{~K}} \\
(\%)\end{array}$ & $\begin{array}{c}\tau_{\mathrm{PL}} \\
(\mathrm{ns})\end{array}$ & $\begin{array}{c}\tau_{\mathrm{r}} \\
(\mathrm{ns})\end{array}$ & $\begin{array}{c}\tau_{\mathrm{nr}} \\
(\mathrm{ns})\end{array}$ \\
\hline Planar SQW & 0.9 & 3.7 & 463 & 3.7 \\
$\phi 2 \mu \mathrm{m}$ & 3.0 & 7.2 & 240 & 7.4 \\
$\phi 1 \mu \mathrm{m}$ & 7.0 & 6.9 & 99 & 7.4 \\
$\phi 500 \mathrm{~nm}$ & 6.0 & 5.3 & 88 & 5.6 \\
\hline \hline
\end{tabular}




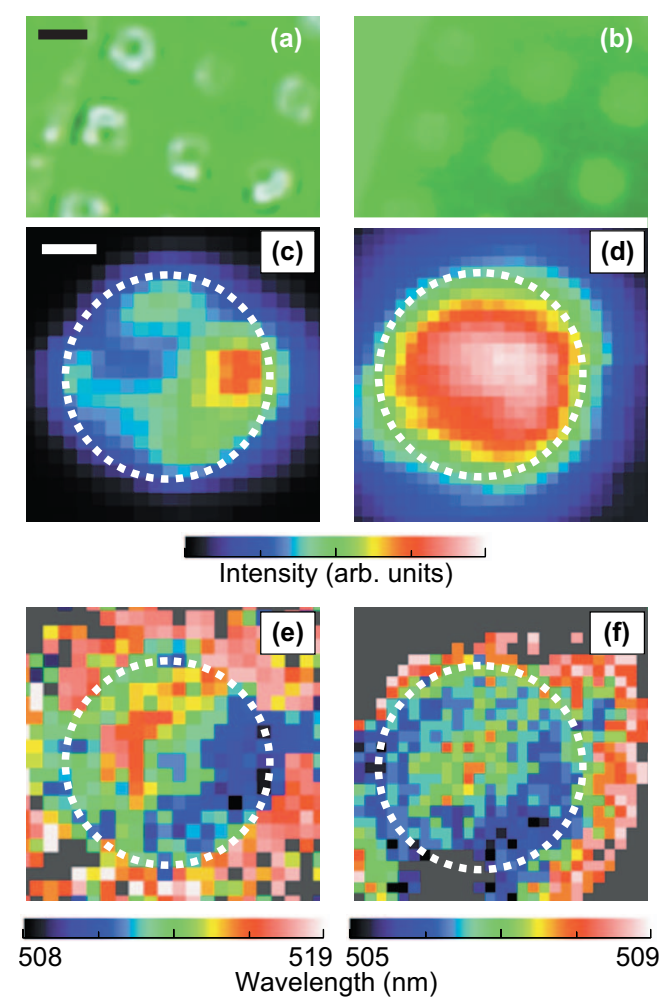

FIG. 7. (Color) (a) fluorescent microscopy image, (c) emission-intensity mapping, and (e) emission-wavelength mapping of $\phi 2 \mu \mathrm{m}$ nanopillars acquired at RT, while (b), (d), and (f) are those acquired at $4.2 \mathrm{~K}$. The scale bar for fluorescent microscopy [(a) and (b)] represents $2 \mu \mathrm{m}$ and that for mappings by confocal microscopy [(c)-(f)] represents $500 \mathrm{~nm}$. The dotted circles in (c)-(f) designate the nanocolumn positions.

those at $4.2 \mathrm{~K}$. Although the reason is unclear, it is likely related to the difference in the dimensionality of the thermally populated excitons for various nanopillars.

On the other hand, the nonradiative recombination lifetime $\left(\tau_{\mathrm{nr}}\right)$ does not clearly depend on the structures, as shown in Table I. Consequently, nanopillar formation improves the internal quantum efficiency. The major concern of nanostructures fabricated by postgrowth processes is surface damages induced by the process. However, current analysis of the nonradiative recombination processes strongly suggests that in the InGaN/GaN nanopillars fabricated by CAIBE, the recombination velocity at the processed surface is negligibly slow compared to other recombination processes.

\section{DOUGHNUT-SHAPE EMISSION PATTERNS}

The doughnut-shape strain distribution revealed in Fig. 6 and radiative/nonradiative recombination processes affect the emission patterns. Figures 7(a) and 7(b) display fluorescent microscopy images, while Figs. 7(c)-7(f) are mappings of emission intensity [Figs. 7(c) and 7(d)] and wavelength [Figs. 7(e) and 7(f)] obtained by confocal microscopy for $\phi 2 \mu \mathrm{m}$ nanopillars. Figures 7(a), 7(c), and 7(e) were acquired at RT, while Figs. 7(b), 7(d), and 7(f) were acquired at 4.2 K. For PL by confocal microscopy, the excitation source was a frequency doubled Ti:sapphire laser emitting at 400 $\mathrm{nm}$ and the spatial resolutions were $500 \mathrm{~nm}$ at RT and 800 $\mathrm{nm}$ at $4.2 \mathrm{~K}$.
The emission wavelengths at RT and $4.2 \mathrm{~K}$ similarly distribute in doughnut shapes, as recognized in Figs. 7(e) and 7(f); the area for longer emission wavelength is surrounded by the area for shorter emission wavelength. Those observations are well accounted for by the doughnut-shape strain distribution [Fig. 6]. That is, the strain relaxation at the side wall reduces QCSE and shortens the emission wavelength. Longer-wavelength emission observed outside the nanopillar is probably due to light scattering within the sample, and the emission intensity is negligibly weak, as supported by Figs. 7(c) and 7(d).

In contrast, the fluorescent microscopy images revealed considerably different emission patterns for RT and $4.2 \mathrm{~K}$ [Figs. 7(a) and 7(b)]; at RT, the emission intensity is distributed in doughnut shapes, whereas at $4.2 \mathrm{~K}$, it is rather uniform in spite of the presence of the strain distribution. Those observations are confirmed by the mappings of single nanopillars by confocal microscopy [Figs. 7(c) and 7(d)]. To understand the difference in the emission patterns at RT and 4.2 $\mathrm{K}$, temperature-dependent physics have to be considered and we found that carrier/exciton recombination processes can well account for the phenomena. The PL intensity, $I_{\mathrm{PL}}$, is determined by competition between radiative and nonradiative recombination processes and can be expressed by $I_{\mathrm{PL}}$ $=G /\left(1+\tau_{\mathrm{r}} / \tau_{\mathrm{nr}}\right)$, where $G$ is the generation rate of carriers/ excitons by laser excitation. This equation tells us that at low temperatures where nonradiative recombination processes are negligible (i.e., $1 / \tau_{\mathrm{nr}} \sim 0$ ), $I_{\mathrm{PL}}$ becomes independent of $\tau_{\mathrm{r}}$. This is consistent with Figs. 7(b) and 7(d). At RT, on the other hand, both $\tau_{\mathrm{r}}$ and $\tau_{\mathrm{nr}}$ may contribute to $I_{\mathrm{PL}}$. As listed in Table I, $\tau_{\mathrm{nr}}$ is not affected by the nanopillars, whereas $\tau_{\mathrm{r}}$ is drastically shortened by smaller nanopillars. Thus, the PL intensity is strengthened around the side wall where the strain is relaxed to shorten $\tau_{\mathrm{r}}$, and the doughnut-shape emission patterns are observed.

\section{RED-EMITTING NANOPILLARS}

In this section, we discuss red-emitting nanopillars with a 4-nm-thick $\operatorname{In}_{0.30} \mathrm{Ga}_{0.70} \mathrm{~N}$ SQW and a 120 -nm-thick GaN capping layer. The purpose to assess these red-emitting nanopillars is twofold; one is to provide supporting data for strain relaxation phenomena in nanopillars using a different structure, and the other is to demonstrate the effect of capping layers on the light extraction.

Figure 8 displays PL spectra of the red-emitting planar SQW, $\phi 2 \mu \mathrm{m}$ and $\phi 500 \mathrm{~nm}$ nanopillars at $4.2 \mathrm{~K}$. The excitation conditions were the same as those for Fig. 3. The emission intensities were normalized by the emitting areas but were significantly structure-dependent, different from those of the green-emitting nanopillars. The cause of this observation is the light extraction, which is discussed later. Concerning the emission peak energy, the blueshift by the nanopillar fabrication suggests the strain relaxation. The degrees of the shift in the red-emitting nanopillars are slightly larger than those in the green-emitting nanopillars, which may be accounted for by a wider well in the red-emitting nanopillars. Although two emission components due to the strain-relaxed and strained regions could not be well re- 


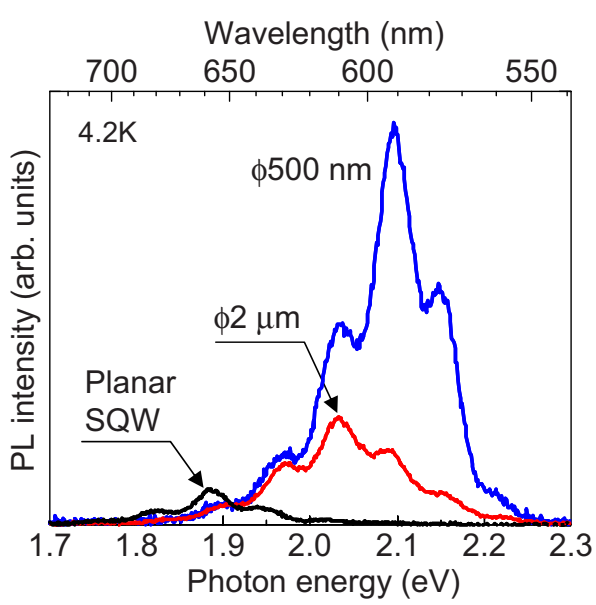

FIG. 8. (Color online) PL spectra of a red-emitting planar SQW and nanopillars with diameters of $2 \mu \mathrm{m}$ and $500 \mathrm{~nm}$ acquired at $4.2 \mathrm{~K}$.

solved, the line widths, which were roughly evaluated from the envelopes of the spectra, increased from $135 \mathrm{meV}$ for the planar SQW to $170 \mathrm{meV}$ for the $\phi 2 \mu \mathrm{m}$ nanopillar, and then, decreased to $135 \mathrm{meV}$ for the $\phi 500 \mathrm{~nm}$ nanopillar. This variation suggests that the $\phi 2 \mu \mathrm{m}$ nanopillar involves multiple components in its spectrum but a larger line width prevented it from being resolved. The presence of multiple components is also supported by the spectrum of the $\phi 500 \mathrm{~nm}$ nanopillar, as the spectrum has a tail on the lower energy side.

TRPL measurements were also performed. The radiative lifetimes at $4.2 \mathrm{~K}$ were drastically shortened from $653 \mathrm{~ns}$ for the planar SQW to 130 and $85 \mathrm{~ns}$ for $\phi 2 \mu \mathrm{m}$ and $\phi 500 \mathrm{~nm}$ nanopillars, respectively. The respective in-plane strains were estimated to be $-3.16 \%,-2.40 \%$, and $-2.18 \%$ and well explain the blueshift of the PL peak positions in Fig. 8 . One of the direct consequences of the shortened radiative lifetimes is improvements in the internal quantum efficiency. Figure 9 shows the Arrhenius plots of the PL integrated intensities. The intensity ratios between RT and $10 \mathrm{~K}$ were $4 \%$, $17 \%$, and $18 \%$ for the planar SQW, $\phi 2 \mu \mathrm{m}$, and $\phi 500 \mathrm{~nm}$ nanopillars, respectively, assuring the improved internal quantum efficiency in the nanopillars.

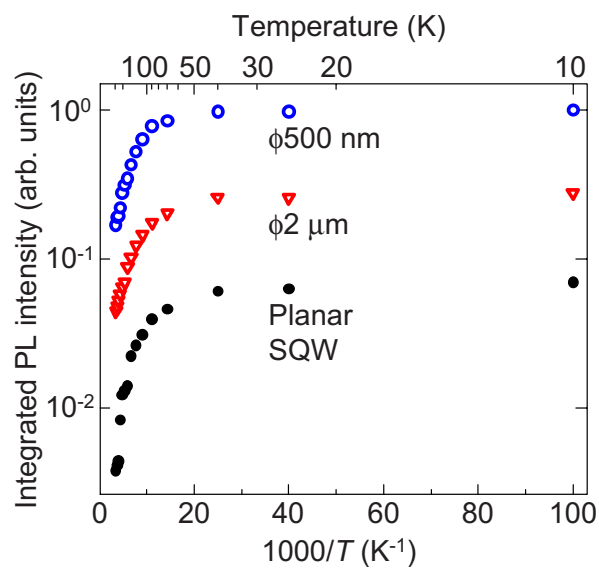

FIG. 9. (Color online) Arrhenius plots of the PL integrated intensities of the red-emitting planar SQW and nanopillars with diameters of $2 \mu \mathrm{m}$ and 500 $\mathrm{nm}$.
Thus, the strain-relaxation related phenomena observed in the red-emitting nanopillars are essentially the same as those for the green-emitting nanopillars, strongly suggesting that the current experimental results and their interpretations can be generalized. On the other hand, the light extraction properties can significantly be affected by the capping layer thickness. As mentioned above, the FDTD simulations have predicted that thicker capping layers enhance light extraction toward the surface normal. In fact, for the green-emitting nanopillars with a $10 \mathrm{~nm}$ capping layer, Fig. 3 demonstrates that the PL intensity at $4.2 \mathrm{~K}$ is rather insensitive to the pillar diameters, suggesting that light extraction is not affected by the pillar fabrication due to the very thin capping layer. In contrast, for the red-emitting nanopillars with a $120 \mathrm{~nm}$ capping layer, the PL intensity normalized by the emitting area increases by factors of 3.8 and 14.3 for $\phi 2 \mu \mathrm{m}$ and $\phi 500 \mathrm{~nm}$ nanopillars, respectively, at $4.2 \mathrm{~K}$, as shown in Figs. 8 and 9. Furthermore, Fig. 9 indicates that these factors remain unchanged up to $50 \mathrm{~K}$. Such temperature independence implies that nonradiative processes are not activated in this low temperature range, and the intensity difference can chiefly be ascribed to the difference in light extraction efficiency. (For more precise evaluation of the light extraction efficiency, an integrating sphere must be used because the current PL setup using an objective lens with a numerical aperture of 0.60 preferentially collect the PL signal normal to the surface.) When the capping layer is thick, smaller nanopillars have stronger PL intensities because nanopillars with a higher aspect ratio can act as better waveguides directing toward the surface normal. Furthermore, fluorescent microscopy images of these red-emitting nanopillars acquired at 4.2 $\mathrm{K}$ confirm the strengthened emission for smaller nanopillars even at low temperatures.

\section{SUMMARY}

In summary, we investigated $\mathrm{InGaN} / \mathrm{GaN}$ nanopillars formed from a planar SQW by CAIBE. It is confirmed that CAIBE does not seriously induce nonradiative recombination centers, which enabled the intrinsic properties of the nanopillars to be extracted. The calculations and the experimental results by PL and TRPL consistently show that the compressive strain in InGaN QWs is relaxed mainly at the free side wall. Consequently, the radiative recombination rate is enhanced at the free side wall by a factor of $4-8$, which indicates that nanopillars can control radiative recombination processes.

\section{ACKNOWLEDGMENTS}

We thank Prof. A. Scherer at CALTECH for the process using CAIBE. One of the authors (K.O.) acknowledges support from JST PRESTO.

${ }^{1}$ Y. Narukawa, M. Sano, M. Ichikawa, S. Minato, T. Sakamoto, T. Yamada, and T. Mukai, Jpn. J. Appl. Phys., Part 2 46, L963 (2007).

${ }^{2}$ A. Kaneta, G. Marutsuki, Y. Narukawa, T. Mukai, K. Okamoto, Y. Kawakami, and S. Fujita, Appl. Phys. Lett. 81, 4353 (2002).

${ }^{3}$ A. Kaneta, T. Mutoh, G. Marutsuki, Y. Narukawa, T. Mukai, Y. Kawakami, and S. Fujita, Appl. Phys. Lett. 83, 3462 (2003).

${ }^{4}$ A. Kaneta, M. Funato, and Y. Kawakami, Phys. Rev. B 78, 125317 (2008).

${ }^{5}$ K. Okamoto, A. Kaneta, Y. Kawakami, S. Fujita, J. Choi, M. Terazima, 
and T. Mukai, J. Appl. Phys. 98, 064503 (2005).

${ }^{6}$ S.-H. Park and S.-L. Chuang, Phys. Rev. B 59, 4725 (1999).

${ }^{7}$ T. Takeuchi, H. Amano, and I. Akasaki, Jpn. J. Appl. Phys., Part 1 39, 413 (2000).

${ }^{8}$ P. Waltereit, O. Brandt, A. Trampert, H. T. Grahn, J. Menniger, M. Ramsteiner, M. Reiche, and K. H. Ploog, Nature (London) 406, 865 (2000).

${ }^{9}$ K. Nishizuka, M. Funato, Y. Kawakami, S. Fujita, Y. Narukawa, and T. Mukai, Appl. Phys. Lett. 85, 3122 (2004).

${ }^{10}$ K. Okamoto, I. Niki, A. Shvartser, Y. Narukawa, T. Mukai, and A. Scherer, Nature Mater. 3, 601 (2004).

${ }^{11}$ M. Yoshizawa, A. Kikuchi, M. Mori, N. Fujita, and K. Kishino, Jpn. J. Appl. Phys., Part 2 36, L459 (1997).

${ }^{12}$ A. Kikuchi, M. Kawai, M. Tada, and K. Kishino, Jpn. J. Appl. Phys., Part 2 43, L1524 (2004).

${ }^{13}$ H. W. Choi, C. W. Jeon, C. Liu, I. M. Watson, M. D. Dawson, P. R. Edwards, R. W. Martin, S. Tripathy, and S. J. Chua, Appl. Phys. Lett. 86 $021101(2005)$
${ }^{14}$ T.-H. Hsueh, H.-W. Huang, C.-C. Kao, Y.-H. Chang, M.-C. Ou-Yang, H.-C. Kuo, and S.-C. Wang, Jpn. J. Appl. Phys., Part 1 44, 2661 (2005).

${ }^{15}$ J. Ristić, C. Rivera, E. Calleja, S. Fernández-Garrido, M. Povoloskyi, and A. Di Carlo, Phys. Rev. B 72, 085330 (2005).

${ }^{16}$ Y. Kawakami, S. Suzuki, A. Kaneta, M. Funato, A. Kikuchi, and K. Kishino, Appl. Phys. Lett. 89, 163124 (2006).

${ }^{17}$ K. Kishino, T. Hoshino, S. Ishizawa, and A. Kikuchi, Electron. Lett. 44, 819 (2008).

${ }^{18}$ H. Sekiguchi, K. Kishino, and A. Kikuchi, Appl. Phys. Express 1, 124002 (2008).

${ }^{19}$ P. Yu, C. H. Shiu, Y.-R. Wu, H. H. Yen, J. R. Chen, C. C. Kao, H.-W. Yang, H. C. Kuo, T. C. Lu, W. Y. Yeh, and S. C. Wang, Appl. Phys. Lett. 93, 081110 (2008).

${ }^{20}$ A. Scherer, J. L. Jewell, Y. H. Lee, J. P. Harbison, and L. T. Florez, Appl. Phys. Lett. 55, 2724 (1989).

${ }^{21}$ M. Funato and Y. Kawakami, J. Appl. Phys. 103, 093501 (2008) 